\title{
Room Temperature Synthesis and Catalytic Properties of Surfactant-Modified Ag Nanoparticles
}

\author{
Weihua Li, Congtao Sun, Baorong Hou, and Xiaodong Zhou \\ Key Laboratory of Corrosion Science, Institute of Oceanology, Chinese Academy of Sciences, Shandong, Qingdao 266071, China \\ Correspondence should be addressed to Xiaodong Zhou, tedzhouqd@hotmail.com
}

Received 27 May 2011; Revised 19 July 2011; Accepted 29 July 2011

Academic Editor: Santanu Bera

Copyright () 2012 Weihua Li et al. This is an open access article distributed under the Creative Commons Attribution License, which permits unrestricted use, distribution, and reproduction in any medium, provided the original work is properly cited.

Well-dispersed Ag nanoparticles with size of 20-30 nm were synthesized in water at room temperature with a self-made novel imidazoline Gemini surfactant quaternary ammonium salt of di (2-heptadecyl-1-formyl aminoethyl imidazoline) hexanediamine. Transmission electron microscopy, X-ray powder diffraction, ultraviolet-visible absorption spectra, and Fourier transform infrared ray were used to characterize the Ag nanoparticles. Results showed that the micellized aggregation of imidazoline Gemini surfactant in water, the growth of $\mathrm{Ag}$ initial particles, and the interaction (adsorption and coordination) between surfactant and $\mathrm{Ag}^{+} / \mathrm{Ag}$ nanoparticles took place simultaneously to form the well-dispersed Ag nanoparticles. Catalytic results show that the surfacemodified Ag product was an active metal catalyst for methyl orange reduction reaction due to the effective adsorption between Ag nanoparticles and methyl orange molecules, which was of promising application in environmental protection.

\section{Introduction}

In recent years, metal nanoparticles have been studied extensively due to their noticeable electrical, optical, and catalytic properties [1-4]. As one of the traditional noble metals, Ag nanoparticles have been synthesized for various applications, such as biomedical antibacterial materials $[5,6]$, catalysis $[1$, 7, 8], tribology [9], and surface-enhanced Raman scattering (SERS) [10-12].

Different methods have been employed to prepare Ag nanoparticles with various morphologies, such as single-source precursor heat treatment at $550^{\circ} \mathrm{C}$ for synthesizing $\mathrm{Ag}$ nanoparticles [13], microwave-assisted oute for Ag nanorods [14] and nanowires [15], microwave-solvothermal synthesis for monodispersed Ag nanoparticles [16], and solvothermal synthesis of chainlike and dendritic Ag nanostructures [17]. Other methods such as photochemical $\gamma$-ray reduction [18], ultraviolet [19], sonochemical [20], and ultrasonic synthesis [21], and so forth have also been exploited recently. However, most of these methods are concerning high-temperature treatment, complicated processing procedures, and exposure to sound or light danger. Thus, exploitation of a facile, energy-efficient, and safe route still remains a challenge.
Room temperature synthesis routes for the fabrication of Ag nanoparticles have been investigated recently, showing promising application feasibility and reliability for largescale manufacture with energy saving and safety. Zhang et al. [5] and Huang et al. [6] prepared Ag nanoparticles at room temperature, showing excellent antibacterial properties; Li et al. [22] prepared Ag nanoparticles at room temperature, which was confirmed to be effective for detecting mercury ion with high sensitivity and selectivity; snowflake-like dendritic Ag Nanostructures [23] and Ag nanowires [24] were also prepared at room temperature. As the concept of "green methods" has become more and more important since the last decade [6], nanomaterials scientists start to pay more attention to facile, safe, and environmentally friendly routes for synthesizing Ag nanoparticles, and room temperature is one of the most promising routes to realize this goal.

Herein, we report a room temperature route to prepare well-dispersed Ag nanoparticles in the presence of a novel imidazoline Gemini surfactant quaternary ammonium salt of di (2-heptadecyl-1-formyl aminoethyl imidazoline) hexanediamine (SHAEIHDI) prepared in our lab [25], which plays multifunctional roles in the whole synthesis procedure of Ag nanoparticles. Catalytic results for reducing methyl 


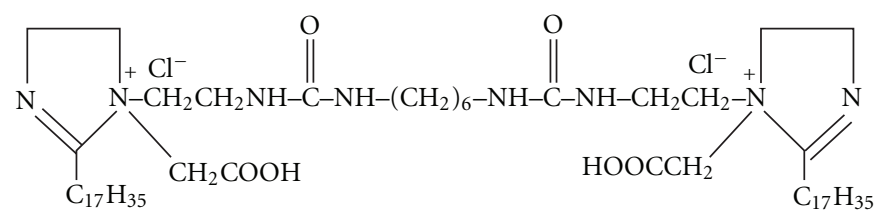

FIgURE 1: Molecular structure of imidazoline Gemini surfactant SHAEIHDI.

orange (MO) by $\mathrm{NaBH}_{4}$ show that the obtained imidazoline Gemini surfactant-modified Ag nanoparticles product is an active, efficient, and promising catalyst for environmental protection.

\section{Experimental}

2.1. Chemicals. All the chemicals, including silver nitrate $\left(\mathrm{AgNO}_{3}\right), 80 \%$ hydrazine hydrate $\left(\mathrm{N}_{2} \mathrm{H}_{4} \cdot \mathrm{H}_{2} \mathrm{O}\right)$, sodium borohydride $\left(\mathrm{NaBH}_{4}\right)$, ethanol, and methyl orange (MO), were of analytical purity and used as received without further purification. The used imidazoline Gemini surfactant quaternary ammonium salt of di (2-heptadecyl-1-formyl aminoethyl imidazoline) hexanediamine (SHAEIHDI) was synthesized according to our previous work [25], and its molecular structure was shown in Figure 1. Water was distilled.

2.2. Preparation and Characterization of Ag Nanoparticles. $0.34 \mathrm{~g}(0.0020 \mathrm{~mol}) \mathrm{AgNO}_{3}$ was dissolved completely into $20 \mathrm{~mL}$ distilled water, and an appropriate amount of SHAEIHDI was dissolved into another $20 \mathrm{~mL}$ distilled water. The two systems were mixed slowly and completely under vigorous stirring to form an orange suspension. Then $1 \mathrm{~mL} 80 \%$ $\mathrm{N}_{2} \mathrm{H}_{4} \cdot \mathrm{H}_{2} \mathrm{O}$ was dropped slowly into the above suspension, which was maintained in dark at room temperature for 24 hours to form black precipitates. The obtained precipitates were washed by distilled water and ethanol for several times, dried in vacuum at $70^{\circ} \mathrm{C}$ for $8 \mathrm{~h}$, and collected carefully for further characterization. The concentration of SHAEIHDI was varied systematically from $0,2,4,6$ to $8 \mathrm{~g} \cdot \mathrm{L}^{-1}$ to investigate its effect on the formation of Ag nanoparticles.

The morphologies and size of the Ag nanoparticles were observed by transmission electron microscopy (TEM) (JEM2000EX Electro-Microscope, Jeol Co., Japan). The Ag products were characterized by X-ray powder diffraction (XRD), using Rigaku (Japan) D/max r-B X-ray diffractometer with $\mathrm{Cu} / \mathrm{K}$ radiation $(\lambda=0.15418 \mathrm{~nm})$ operating at $40 \mathrm{kV}$ and $30 \mathrm{~mA}$ with $2 \theta$ ranging from $10^{\circ}$ to $80^{\circ}$. The samples were also measured by ultraviolet-visible absorption spectra (UVvis) (756CRT UV-vis spectrophotometer, Shanghai Precision and Scientific Instrument Co. Ltd., China) and infrared ray (IR) (510P Fourier transform infrared spectrophotometer, Nicolet, USA).

2.3. Catalytic Properties of Ag Nanoparticles. $0.005 \mathrm{~g} \mathrm{Ag}$ nanoparticles and $0.02 \mathrm{~g} \mathrm{NaBH}_{4}$ were added into $20 \mathrm{~mL} 1 \times$ $10^{-5} \mathrm{~mol} \cdot \mathrm{L}^{-1} \mathrm{MO}$ solution, and the system was mixed under stirring. The change in color of the system could be indicated by detecting the absorbance variation at the wavelength of the absorbance maximum $\left(\lambda_{\max }=463 \mathrm{~nm}\right)$ of $\mathrm{MO}$ using the Varian Cary 50 UV-vis spectrophotometer (Varian Co., Australia).

\section{Results and Discussion}

3.1. Characterization of $\mathrm{Ag}$ Samples. Figure 2 shows the typical TEM images and SAED pattern of Ag samples prepared with varied SHAEIHDI concentration. It can be seen from Figure 2(a) that only irregular Ag agglomerates with serious aggregation can be obtained without SHAEIHDI. When $2 \mathrm{~g} \cdot \mathrm{L}^{-1}$ SHAEIHDI is used as shown in Figure 2(b), Ag nanoparticles with diameter of $50-100 \mathrm{~nm}$ have been obtained with improved dispersion. Further increasing the surfactant concentration to $4 \mathrm{~g} \cdot \mathrm{L}^{-1}$ shown in Figure 2(c) can give rise to the formation of better-dispersed $\mathrm{Ag}$ nanoparticles with diameter of 30-50 nm. Figures 2(d) and 2(e) show the TEM image and SAED pattern of Ag nanoparticles prepared with $6 \mathrm{~g} \cdot \mathrm{L}^{-1}$ SHAEIHDI, indicating that welldispersed Ag nanoparticles product with uniform size of 20 $30 \mathrm{~nm}$ has been obtained, which is of single-crystal structure. When the SHAEIHDI concentration is increased up to $8 \mathrm{~g} \cdot \mathrm{L}^{-1}$ as shown in Figure 2(f), the size of Ag nanoparticles becomes larger $(30-80 \mathrm{~nm})$ and not uniform. Thus, $6 \mathrm{~g} \cdot \mathrm{L}^{-1}$ is the optimum concentration for the best dispersion of $\mathrm{Ag}$ nanoparticles with uniform size.

The XRD pattern of Ag nanoparticles with $6 \mathrm{~g} \cdot \mathrm{L}^{-1}$ SHAEIHDI is shown in Figure 3. The intensive diffraction peaks can be indexed to the cubic Ag nanocrystals (JCPDS file no. 04-0783), corresponding to its plane of (111), (200), and (220). The sharp diffraction peaks indicate the relatively high crystallinity of the obtained Ag nanoparticles product. In addition, the two small peaks at around $19^{\circ}$ and $28^{\circ}$ could come from orthorhombic $\mathrm{AgNO}_{3}$ crystalline (JCPDS file no. 06-0363), and the peak at about $32^{\circ}$ could be attributed to hexagonal $\mathrm{Ag}_{2} \mathrm{O}$ crystals (JCPDS File No. 42-0874) [4]. These results might be resulted from the washing procedures in this room temperature synthesis route, as well as the confinement effect [4].

Figure 4 showed the FT-IR spectra of SHAEIHDI (a) and $6 \mathrm{~g} \cdot \mathrm{L}^{-1}$ SHAEIHDI-modified Ag nanoparticles (b). In Figure 4(a)), the absorption peak at $3315 \mathrm{~cm}^{-1}$ was attributed to the stretching vibration of $\mathrm{N}-\mathrm{H}$, and the peaks at $2919 \sim 2851 \mathrm{~cm}^{-1}$ were attributed to the aliphatic $\mathrm{C}-\mathrm{H}$ vibrations in the long-chain alkyl $\left(-\mathrm{C}_{17} \mathrm{H}_{35}\right)$. The absorbance peak at $1660 \mathrm{~cm}^{-1}$ was attributed to $-\mathrm{NH}-\mathrm{C}=\mathrm{O}$. The absorbance of imidazoline ring and $\mathrm{C}=\mathrm{O}$ in carboxyl were revealed at $1604 \mathrm{~cm}^{-1}$ and $1746 \mathrm{~cm}^{-1}$, respectively. The typical peaks of SHAEIHDI also existed in Figure 4(b), indicating that a little amount of SHAEIHDI was adsorbed 


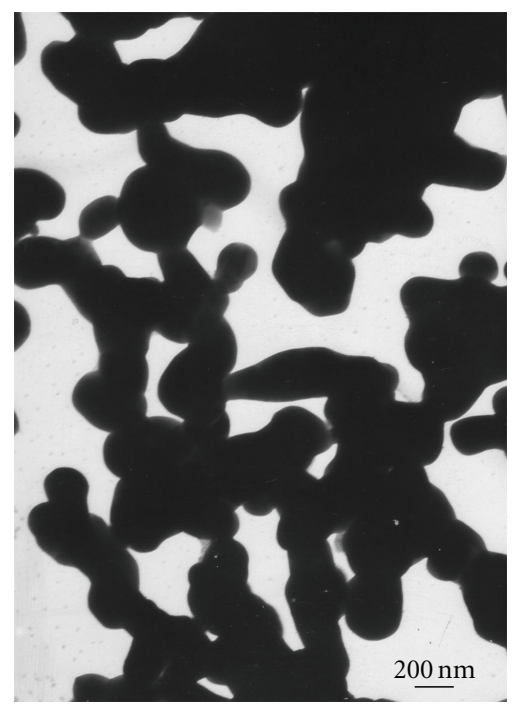

(a)

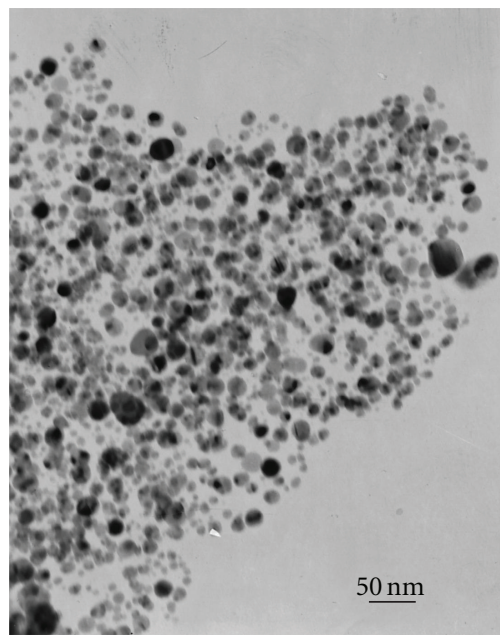

(d)

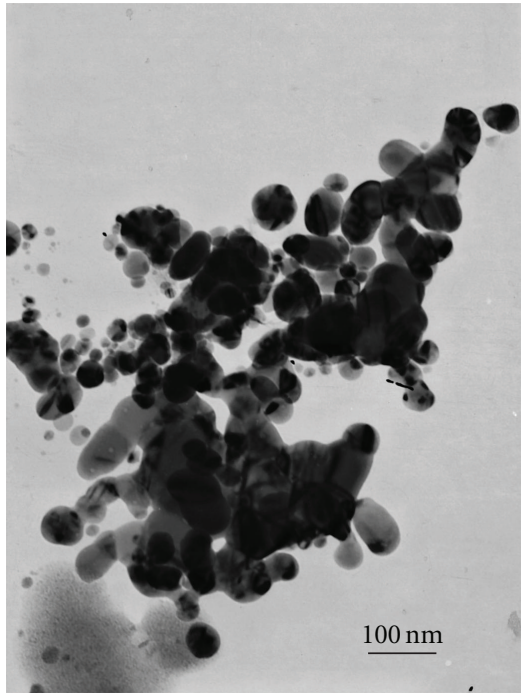

(b)

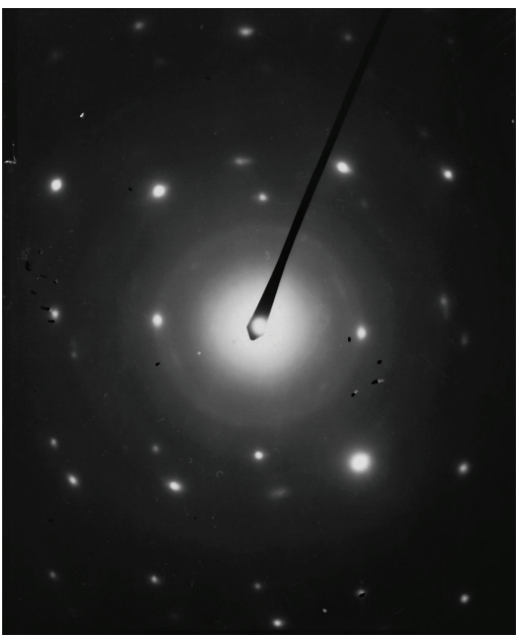

(e)

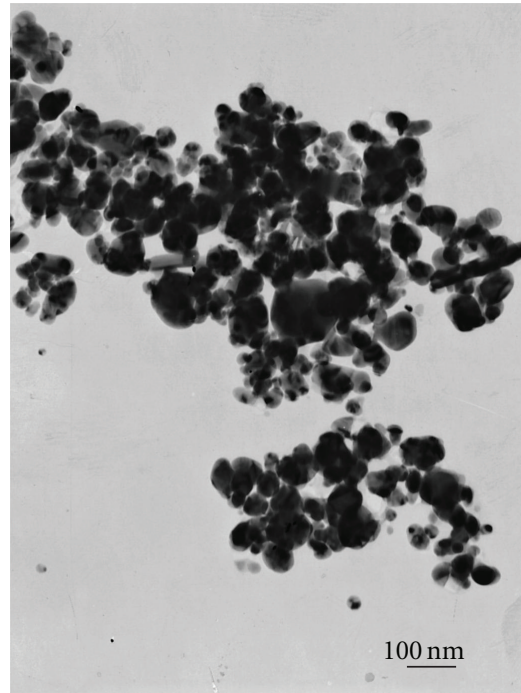

(c)

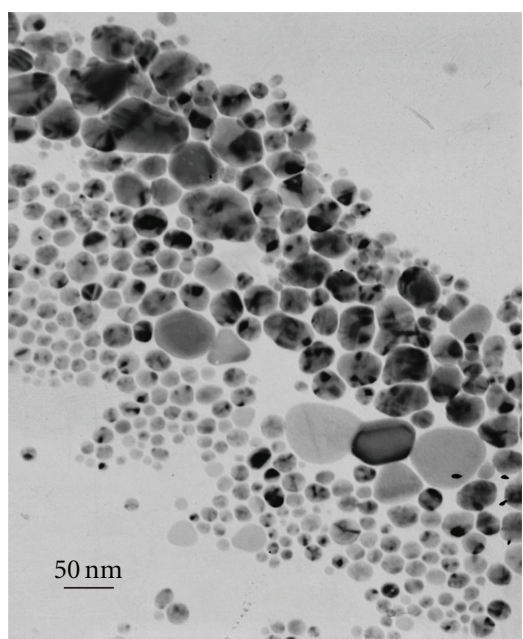

(f)

FIGURE 2: TEM images and SAED pattern of Ag samples prepared in water at room temperature with varied SHAEIHDI concentration: (a) $0 \mathrm{~g} \cdot \mathrm{L}^{-1}$; (b) $2 \mathrm{~g} \cdot \mathrm{L}^{-1} ;$ (c) $4 \mathrm{~g} \cdot \mathrm{L}^{-1} ;(\mathrm{d}-\mathrm{e}) 6 \mathrm{~g} \cdot \mathrm{L}^{-1} ;$ (f) $8 \mathrm{~g} \cdot \mathrm{L}^{-1}$.

onto the surface of Ag nanoparticles to form a modification layer and could not be washed off totally by ethanol and distilled water. Moreover, the absorbance of $-\mathrm{NH}-\mathrm{C}=\mathrm{O}$ at $1660 \mathrm{~cm}^{-1}$ in Figure 4(a) weakened obviously in Figure 4(b), showing that there was a strong coordination effect between $\mathrm{C}=\mathrm{O}$ bond in SHAEIHDI and Ag nanoparticles, which is in good agreement with the color change in the preparation Section 2.2 of Ag nanoparticles, indicating that SHAEIHDI is not only a surface-modification agent to Ag nanoparticles, but a coordination agent as well.

Figure 5 showed the UV-vis spectra of SHAEIHDI (a) and $6 \mathrm{~g} \cdot \mathrm{L}^{-1}$ SHAEIHDI-modified Ag nanoparticles (b). The two peaks appeared at 200 and $240 \mathrm{~nm}$ in Figure 5(a) corresponded to the absorption of imidazoline ring and conjugated $\mathrm{C}=\mathrm{O}$, respectively. In Figure 5(b), the absorbance of imidazoline ring still existed, but the absorption of $\mathrm{C}=\mathrm{O}$ at $240 \mathrm{~nm}$ in Figure 5(a) weakened in Figure 5(b), showing that there was a strong coordination effect between $\mathrm{C}=\mathrm{O}$ and $\mathrm{Ag}$ nanoparticles, which could give rise to the good stability and dispersion capacity of the prepared $\mathrm{Ag}$ nanoparticles in both organic and inorganic medium. The wide peak appeared at around $275 \mathrm{~nm}$ corresponded to the absorbance of Ag nanoparticles [26]. Interestingly, the surface plasmon resonance (SPR) band at round $400 \mathrm{~nm}$ $[26,27]$ is weak and not very obvious in Figure 5(b), which might be caused by this novel imidazoline Gemini surfactant-assisted room temperature synthesis route, and the exact reason deserves further investigation. The UVvis analysis can further confirm the good surface-modifying effect of SHAEIHDI on Ag nanoparticles, which is in good accordance with the FT-IR results.

3.2. Formation of $A g$ Nanoparticles. In the preparation process of Ag nanoparticles, imidazoline Gemini surfactant 


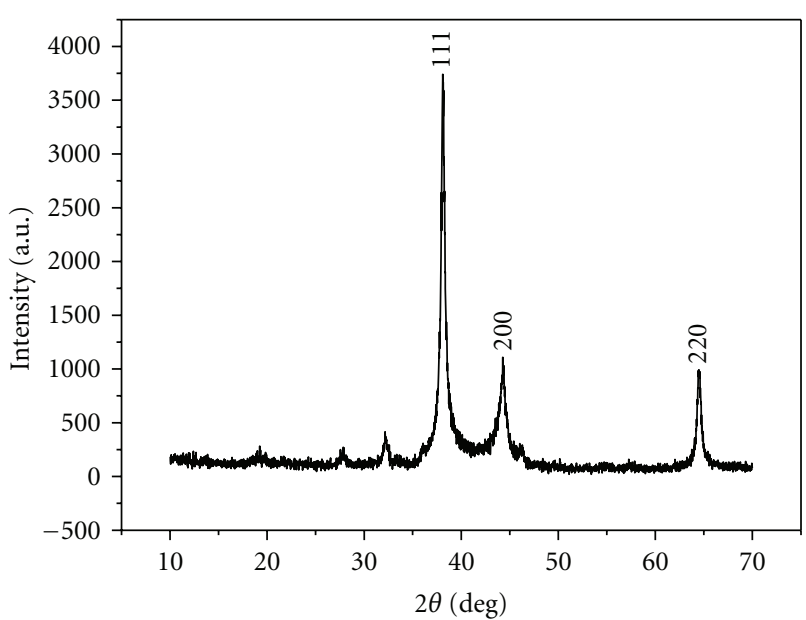

FIGURE 3: XRD pattern of Ag nanoparticles prepared with $6 \mathrm{~g} \cdot \mathrm{L}^{-1}$ SHAEIHDI at room temperature.

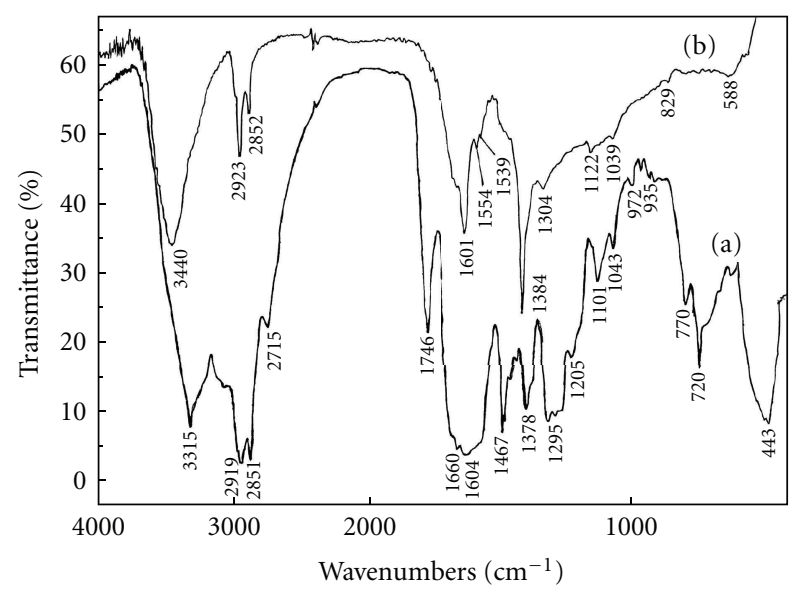

FIGURE 4: FT-IR spectra of SHAEIHDI (a) and Ag nanoparticles (b).

SHAEIHDI is served as not only surface-modification agent, but also a coordinating agent, which can be confirmed by the color change (orange) after mixing SHAEIHDI and $\mathrm{AgNO}_{3}$ solutions. Furthermore, the FT-IR and UV-vis measurements can also proof that SHAEIHDI can coordinate with $\mathrm{Ag}^{+}$ to form a SHAEIHDI- $\mathrm{Ag}^{+}$complex. During the growth of $\mathrm{Ag}$ nanoparticles, the long-chain alkyls $\left(-\mathrm{C}_{17} \mathrm{H}_{35}\right)$ in SHAEIHDI (its formula structure is shown in Figure 1) have excellent steric effects, which can stop the new-formed $\mathrm{Ag}$ nanostructures from aggregating and make them stabilize in aqueous medium. The formation speed of Ag nanoparticles can also be controlled by the SHAEIHDI adsorbed on the surface of $\mathrm{Ag}$ nanoparticles and thus prevent them from aggregating as well. The special micellization behavior of SHAEIHDI molecules in water and the interaction between SHAEIHDI molecules and Ag nanoparticles take an active part in the whole procedure.

In our previous studies, an imidazoline surfactant quaternary ammonium salt of 2-undecyl-1-dithioureido-ethylimidazoline (SUDEI) has been synthesized [28] and used

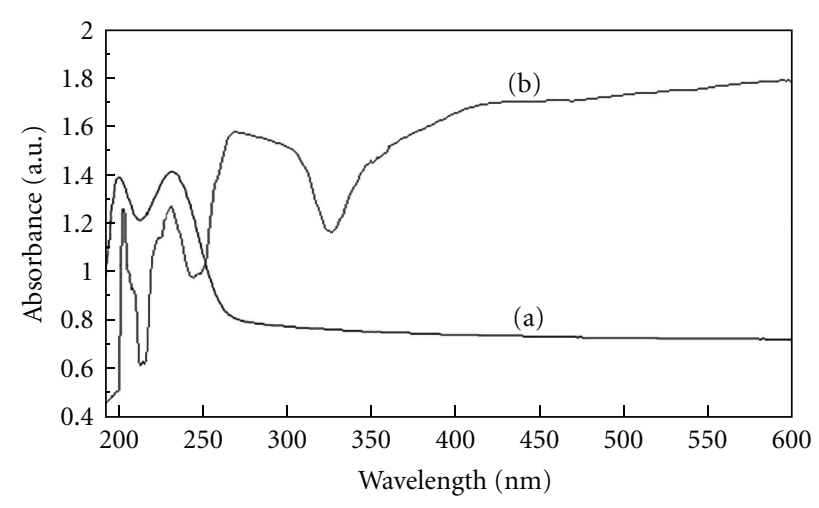

FIGURE 5: UV-vis spectra of SHAEIHDI (a) and Ag nanoparticles (b).

as the surface-modifying agent for preparing nanomaterials, and its sphere-like micelles in water have been confirmed $[29,30]$. Imidazoline Gemini surfactant SHAEIHDI has the similar molecular structure to SUDEI but with more active elements $\mathrm{N}$ and $\mathrm{O}$, which can also form sphere-like micelles in water, and Ag nanoparticles will grow along the hydrophilic groups in the micelles. The micellized aggregation of SHAEIHDI in water, the growth of Ag initial particles, and the adsorption and coordination of SHAEIHDI on $\mathrm{Ag}^{+}$ and Ag nanoparticles will take place at the same time to form the final well-dispersed $\mathrm{Ag}$ nanoparticles with narrow size distribution [31, 32].

In addition, an appropriate concentration of SHAEIHDI will also play a vital role in the formation of well-dispersed Ag nanoparticles. When $2 \mathrm{~g} \cdot \mathrm{L}^{-1}$ SHAEIHDI is used (see Figure 2(b)), the aggregation of SHAEIHDI is not enough, and sphere-like micelles cannot form effectively, thus only Ag nanoparticles with size of 50-100 nm can be formed, and the aggregation is obvious. Further increasing the SHAEIHDI concentration to $4 \mathrm{~g} \cdot \mathrm{L}^{-1}$ can improve the dispersion of $\mathrm{Ag}$ nanoparticles, and the size can be further tailored to $30-$ $50 \mathrm{~nm}$ (see Figure 2(c)), indicating that the micelles of SHAEIHDI are taking more effective role in the formation of Ag nanoparticles. When $6 \mathrm{~g} \cdot \mathrm{L}^{-1}$ SHAEIHDI is employed, SHAEIHDI will aggregate effectively to form perfect spherelike micelles, giving rise to the successful formation of welldispersed Ag nanoparticles with narrow size of $20-30 \mathrm{~nm}$ (see Figure $2(\mathrm{~d}))$. However, if more SHAEIHDI $\left(8 \mathrm{~g} \cdot \mathrm{L}^{-1}\right)$ is used as shown in Figure 2(f), SHAEIHDI molecules will aggregate excessively to form larger micelles, which can lead to the formation of larger-sized Ag nanoparticles (30-80 nm) with aggregation to a certain degree. Moreover, the formed Ag nanoparticles are stable in aqueous medium and can be dispersed easily in water without obvious precipitation, which is important for the later catalytic experiments. However, the Ag particles are not monodispersed as shown in all of the above samples prepared with varied surfactant concentrations, and more work should be done to further tailor the particle size to a desirable and monodispersed range for potential applications.

In a word, an appropriate amount of SHAEIHDI is necessary and vital for the successful formation of well-dispersed 


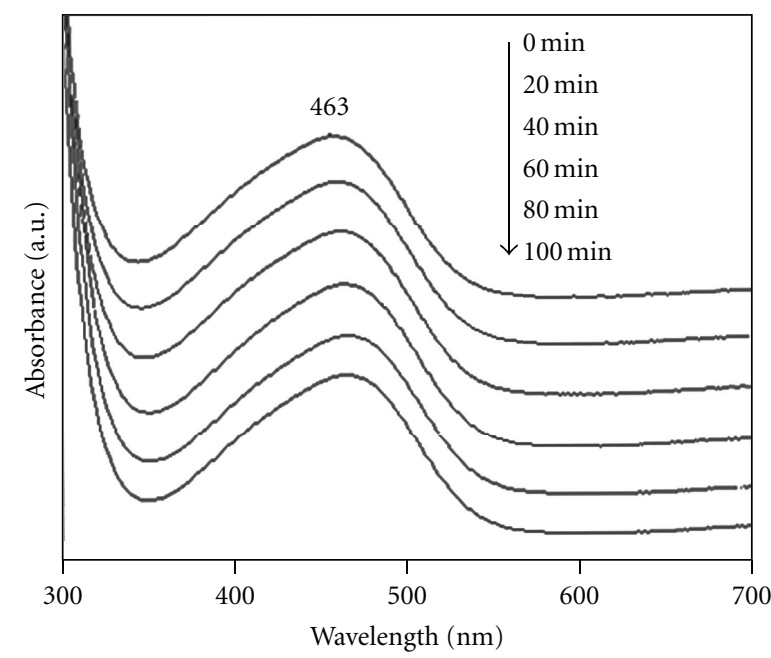

(a)

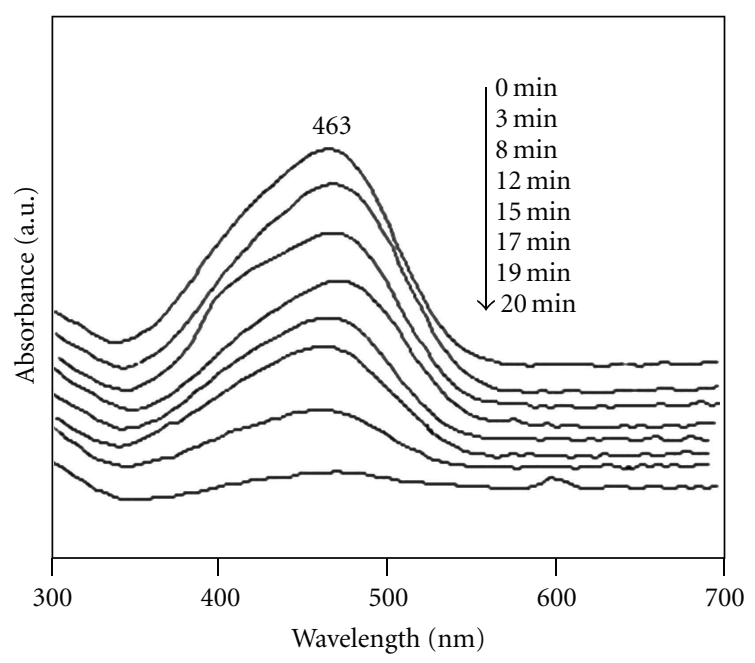

(b)

Figure 6: UV-vis spectra of $\mathrm{MO}+\mathrm{NaBH}_{4}$ solution at different irradiation time. (a) without Ag; (b) with Ag nanoparticles.

Ag nanoparticles. The surfactant SHAEIHDI prepared in our lab is served as surface-modifying agent, coordinating agent, morphology-controlling agent, and stabilizing agent for the formation of well-dispersed Ag nanoparticles. This imidazoline Gemini surfactant and the facile room temperature process could be extended to the synthesis of other metal nanoparticles with potential applications.

3.3. Catalytic Activity of Ag Nanoparticles. Ag nanoparticles sample with diameter of 20-30 nm (Figure 2(d)) was used to test its catalytic properties for the reaction of $\mathrm{MO}$ reduced by $\mathrm{NaBH}_{4}$. The Ag nanoparticles can be dispersed very well in the MO solution system with good stability due to the excellent surface-modifying and stabilizing effects of surfactant SHAEIHDI.

The catalytic results of Ag nanoparticles were shown in Figure 6. It can be seen from Figure 6(a) that the blank experiments performed without any Ag catalysts show nearly no absorbance change of MO solution at its $\lambda_{\max }$, indicating that $\mathrm{MO}$ is not reduced effectively by $\mathrm{NaBH}_{4}$, or the reduction rate is very slow. However, when Ag nanoparticles were added as shown in Figure 6(b), the absorbance of MO solution decreased quickly with reaction time to almost none within the last 3 minutes (17-20 min), indicating that MO was reduced effectively by $\mathrm{NaBH}_{4}$ under the catalytic effect of Ag nanoparticles.

Today environmental protection has become more and more important for human beings, and some toxic and stable dye molecules like $\mathrm{MO}$ are dangerous to the environment [33]. MO is an organic sulfosalt dye, which can be reduced by reductants like $\mathrm{NaBH}_{4}$ to form small organic molecules firstly and nontoxic species finally, but the reduction rate is very slow. Metal nanomaterials with high reactive activity and specific surface area can accelerate the reduction rate of dyes, thus increasing the reducing efficiency. Chen et al. [34] prepared PS/Ni nanocomposite spheres, showing noticeable catalytic performance for reducing methylene blue trihydrate (MBt) by $\mathrm{NaBH}_{4}$; Saha et al. [1] prepared Ag nonoparticles, which is proved to be a very effective catalyst for reducing 4nitrophenol to 4 -aminophenol by $\mathrm{NaBH}_{4}$, and adsorption of the reactants on the surface of the catalyst can mainly account for the catalytic mechanism.

In this paper, after adding $\mathrm{Ag}$ nanoparticles into the $\mathrm{MO}$ and $\mathrm{NaBH}_{4}$ solution as the catalyst, adsorption can take place between the well-dispersed Ag nanoparticles and $\mathrm{MO}$ molecules. The large specific surface area of Ag nanoparticles can help increase the specific surface area of MO, thus increasing its reactive activity. The modification layer of SHAEIHDI on the surface of $\mathrm{Ag}$ nanoparticles can make them stable with good dispersion in the MO aqueous system, which can also promote the effective adsorption between Ag nanoparticles and MO molecules. As a consequence, the oxidation-reduction reaction between the active MO and $\mathrm{NaBH}_{4}$ can happen more easily, effectively, and faster, indicating that the Ag nanoparticles product is an active and promising catalyst for environmental protection.

\section{Conclusion}

A facile room temperature synthesis route has been presented for preparing well-dispersed Ag nanoparticles with uniform size of $20-30 \mathrm{~nm}$ in the presence of imidazoline Gemini surfactant SHAEIHDI prepared in our lab. Characterization results show that SHAEIHDI plays multifunctional roles in the whole formation procedure of Ag nanoparticles, such as surface-modifying agent, coordinating agent, morphologycontrolling agent, and stabilizing agent. The SHAEIHDImodified Ag nanoparticles sample shows excellent catalytic performance for reducing $\mathrm{MO}$ by $\mathrm{NaBH}_{4}$, indicating that the obtained surface-modified Ag nanoparticles product is a potential and promising catalyst for environmental protection. The imidazoline Gemini surfactant SHAEIHDI 
and the facile room temperature synthesis route could be extended to the preparation of other metal nanoparticles for various applications.

\section{Acknowledgments}

The authors gratefully acknowledge the support of Grant P2010-1-ZH-005 from Shinan Scientific and Technological R\&D Foundation of Qingdao City, China, Grant BS2009 HZ002 from Ph.D. Foundation of Shandong Province, China, and K. C. Wong Education Foundation, Hong Kong.

\section{References}

[1] S. Saha, A. Pal, S. Kundu, S. Basu, and T. Pal, "Photochemical green synthesis of calcium-alginate-stabilized $\mathrm{Ag}$ and $\mathrm{Au}$ nanoparticles and their catalytic application to 4-nitrophenol reduction," Langmuir, vol. 26, no. 4, pp. 2885-2893, 2010.

[2] C. J. Murphy, A. M. Gole, J. W. Stone et al., "Gold nanoparticles in biology: beyond toxicity to cellular imaging," Accounts of Chemical Research, vol. 41, no. 12, pp. 1721-1730, 2008.

[3] N. L. Rosi and C. A. Mirkin, "Nanostructures in biodiagnostics," Chemical Reviews, vol. 105, no. 4, pp. 1547-1562, 2005.

[4] C. Faure, A. Derré, and W. Neri, "Spontaneous formation of silver nanoparticles in multilamellar vesicles," Journal of Physical Chemistry B, vol. 107, no. 20, pp. 4738-4746, 2003.

[5] Y. W. Zhang, H. S. Peng, W. Huang, Y. F. Zhou, and D. Y. Yan, "Facile preparation and characterization of highly antimicrobial colloid Ag or Au nanoparticles," Journal of Colloid and Interface Science, vol. 325, no. 2, pp. 371-376, 2008.

[6] N. M. Huang, H. N. Lim, S. Radiman et al., "Sucrose ester micellar-mediated synthesis of Ag nanoparticles and the antibacterial properties," Colloids and Surfaces A, vol. 353, no. 1, pp. 69-76, 2010.

[7] L. Y. Li and D. L. King, "Synthesis and characterization of silver hollandite and its application in emission control," Chemistry of Materials, vol. 17, no. 17, pp. 4335-4343, 2005.

[8] C. Burda, X. B. Chen, R. Narayanan, and M. A. ElSayed, "Chemistry and properties of nanocrystals of different shapes," Chemical Reviews, vol. 105, no. 4, pp. 1025-1102, 2005.

[9] L. Sun, X. J. Tao, Y. B. Zhao, and Z. J. Zhang, "Synthesis and tribology properties of stearate-coated Ag nanoparticles," Tribology Transactions, vol. 53, no. 2, pp. 174-178, 2010.

[10] K. Kim, H. K. Park, and N. H. Kim, "Silver-particlebased surface-enhanced Raman scattering spectroscopy for biomolecular sensing and recognition," Langmuir, vol. 22, no. 7, pp. 3421-3427, 2006.

[11] L. H. Lu, A. Kobayashi, K. Tawa, and Y. Ozaki, "Silver nanoplates with special shapes: controlled synthesis and their surface plasmon resonance and surface-enhanced Raman scattering properties," Chemistry of Materials, vol. 18, no. 20, pp. 4894-4901, 2006.

[12] Y. Yang, S. Matsubara, L. M. Xiong, T. Hayakawa, and M. Nogami, "Solvothermal synthesis of multiple shapes of silver nanoparticles and their SERS properties," Journal of Physical Chemistry C, vol. 111, no. 26, pp. 9095-9104, 2007.

[13] A. Pramanik and G. Das, "Precursory Ag-bipyridine 2D coordination polymer: a new and efficient route for the synthesis of Ag nanoparticles," CrystEngComm, vol. 12, no. 2, pp. 401-405, 2010.

[14] M. N. Nadagouda and R. S. Varma, "Microwave-assisted shape-controlled bulk synthesis of $\mathrm{Ag}$ and Fe nanorods in poly(ethylene glycol) solutions," Crystal Growth \& Design, vol. 8, no. 1, pp. 291-295, 2008.

[15] L. F. Gou, M. Chipara, and J. M. Zaleski, "Convenient, rapid synthesis of Ag nanowires," Chemistry of Materials, vol. 19, no. 7, pp. 1755-1760, 2007.

[16] F. Gao, Q. Y. Lu, and S. Komarneni, "Interface reaction for the self-assembly of silver nanocrystals under microwave-assisted solvothermal conditions," Chemistry of Materials, vol. 17, no. 4, pp. 856-860, 2005.

[17] G. D. Wei, C. W. Nan, Y. Deng, and Y. H. Lin, "Formation and accumulation of $\alpha$-acids, $\beta$-acids, desmethylxanthohumol, and xanthohumol during flowering of hops (Humulus lupulus L.)," Chemistry of Materials, vol. 51, no. 15, pp. 4436-4441, 2003.

[18] A. Torreggiani, Z. Jurasekova, M. D’Angelantonio, M. Tamba, J. V. Garcia-Ramos, and S. Sanchez-Cortes, "Fabrication of Ag nanoparticles by $\gamma$-irradiation: application to surfaceenhanced Raman spectroscopy of fungicides," Colloids and Surfaces A, vol. 339, no. 1-3, pp. 60-67, 2009.

[19] M. Harada, K. Saijo, and N. Sakamoto, "Characterization of metal nanoparticles prepared by photoreduction in aqueous solutions of various surfactants using UV-vis, EXAFS and SAXS," Colloids and Surfaces A, vol. 349, no. 1-3, pp. 176-188, 2009.

[20] L. P. Jiang, A. N. Wang, Y. Zhao, J. R. Zhang, and J. J. Zhu, "A novel route for the preparation of monodisperse silver nanoparticles via a pulsed sonoelectrochemical technique," Inorganic Chemistry Communications, vol. 7, no. 4, pp. 506$509,2004$.

[21] X. K. Wang, L. Shao, W. L. Guo, J. G. Wang, Y. P. Zhu, and C. Wang, "Synthesis of dendritic silver nanostructures by means of ultrasonic irradiation," Ultrasonics Sonochemistry, vol. 16, no. 6, pp. 747-751, 2009.

[22] W. B. Li, Y. Y. Guo, K. McGill, and P. Zhang, "A facile synthesis of $\mathrm{Ag}$ nanoparticles for mercury ion detection with high sensitivity and selectivity," New Journal of Chemistry, vol. 34, no. 6, pp. 1148-1152, 2010.

[23] X. P. Sun and M. Hagner, "Novel preparation of snowflake-like dendritic nanostructures of $\mathrm{Ag}$ or Au at room temperature via a wet-chemical route," Langmuir, vol. 23, no. 18, pp. 91479150, 2007.

[24] D. B. Zhang, L. M. Qi, J. H. Yang, J. M. Ma, H. M. Cheng, and L. Huang, "Wet chemical synthesis of silver nanowire thin films at ambient temperature," Chemistry of Materials, vol. 16, no. 5, pp. 872-876, 2004.

[25] W. H. Li, H. W. Tian, and X. D. Zhou, "Tenside Surfactants Detergents," 2011 (submitted).

[26] P. K. Khanna, N. Singh, D. Kulkarni, S. Deshmukh, S. Charan, and P. V. Adhyapak, "Water based simple synthesis of redispersible silver nano-particles," Materials Letters, vol. 61, no. 16, pp. 3366-3370, 2007.

[27] E. Hutter, J. H. Fendler, and D. Roy, "Surface plasmon resonance studies of gold and silver nanoparticles linked to gold and silver substrates by 2-aminoethanethiol and 1,6hexanedithiol," Journal of Physical Chemistry B, vol. 105, no. 45, pp. 11159-11168, 2001.

[28] X. D. Zhou, H. Q. Shi, X. Fu, X. F. Zhao, and Z. S. Hu, "Synthesis and interfacial properties of imidazoline surfactants," Journal of Dispersion Science and Technology, vol. 28, no. 7, pp. 1086-1092, 2007.

[29] D. M. Wu, X. D. Zhou, X. Fu, and H. Q. Shi, "Synthesis and characterization of molybdenum disulfide micro-sized spheres," Journal of Materials Science, vol. 41, no. 17, pp. 56825686, 2006. 
[30] X. D. Zhou, H. Q. Shi, X. Fu, H. Liu, X. Zhao, and Z. S. Hu, "Facile fabrication of surfactant-modified $\mathrm{ZnS}$ hollow spheres at room temperature and its tribological properties in liquid paraffin," Journal of Dispersion Science and Technology, vol. 29, no. 2, pp. 250-256, 2008.

[31] Z. S. Hu, L. Y. Li, X. D. Zhou, X. Fu, and G. H. Gu, "Solvothermal synthesis of hollow ZnS spheres," Journal of Colloid and Interface Science, vol. 294, no. 2, pp. 328-333, 2006.

[32] X. D. Zhou, H. Q. Shi, D. M. Huang, S. M. Jia, X. Fu, and K. Jiao, "Room temperature synthesis and electrochemical application of imidazoline surfactant-modified Ag2S nanocrystals," Materials Letters, vol. 62, no. 16, pp. 2407-2410, 2008.

[33] H. Q. Wang, G. H. Li, L. C. Jia, G. Z. Wang, and C. J. Tang, "Controllable preferential-etching synthesis and photocatalytic activity of porous $\mathrm{ZnO}$ nanotubes," Journal of Physical Chemistry C, vol. 112, no. 31, pp. 11738-11743, 2008.

[34] M. Chen, J. Zhou, L. Xie, G. X. Gu, and L. M. Wu, "Facile fabrication method of PS/Ni nanocomposite spheres and their catalytic property," Journal of Physical Chemistry C, vol. 111, no. 32, pp. 11829-11835, 2007. 


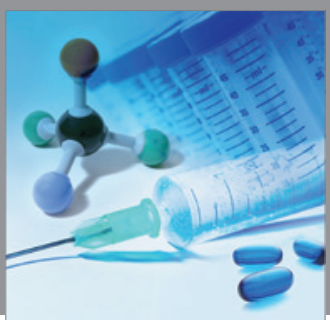

International Journal of

Medicinal Chemistry

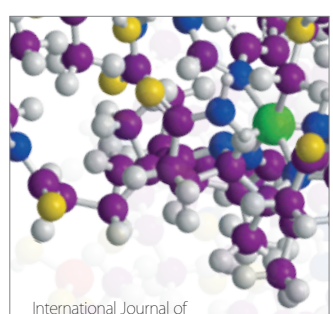

Carbohydrate Chemistry

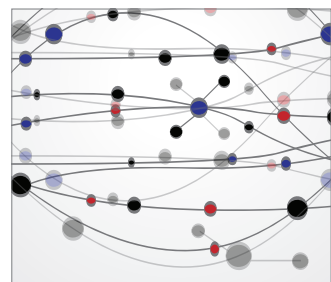

The Scientific World Journal
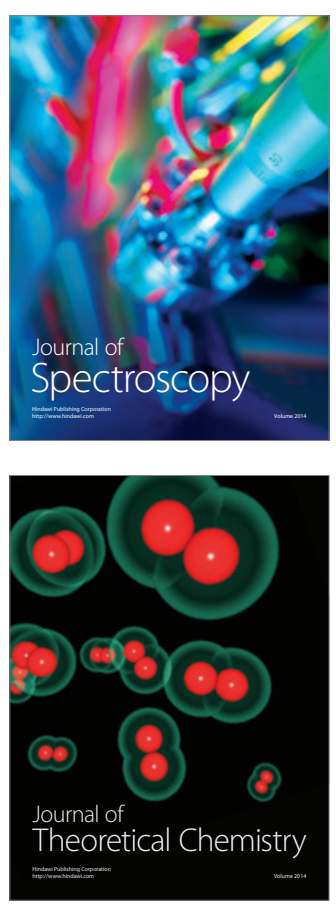
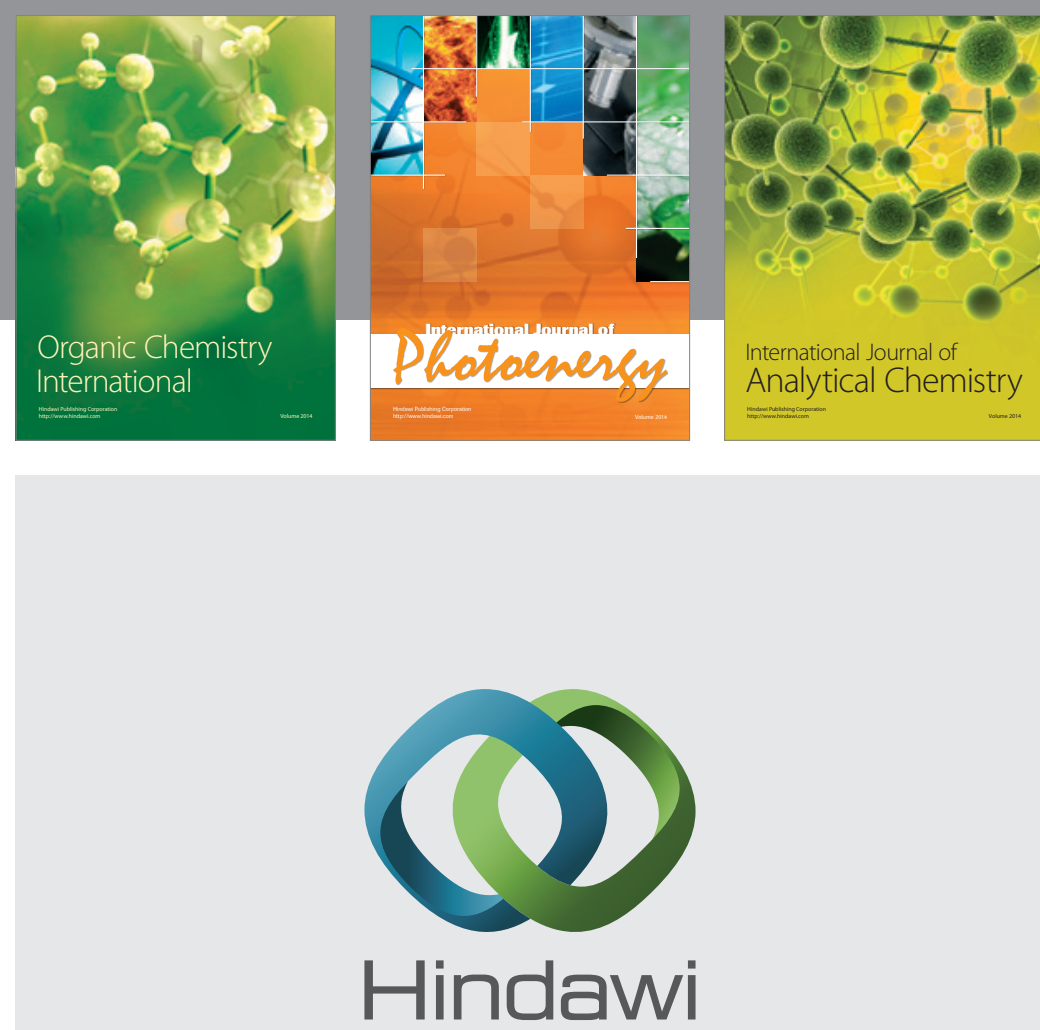

Submit your manuscripts at

http://www.hindawi.com
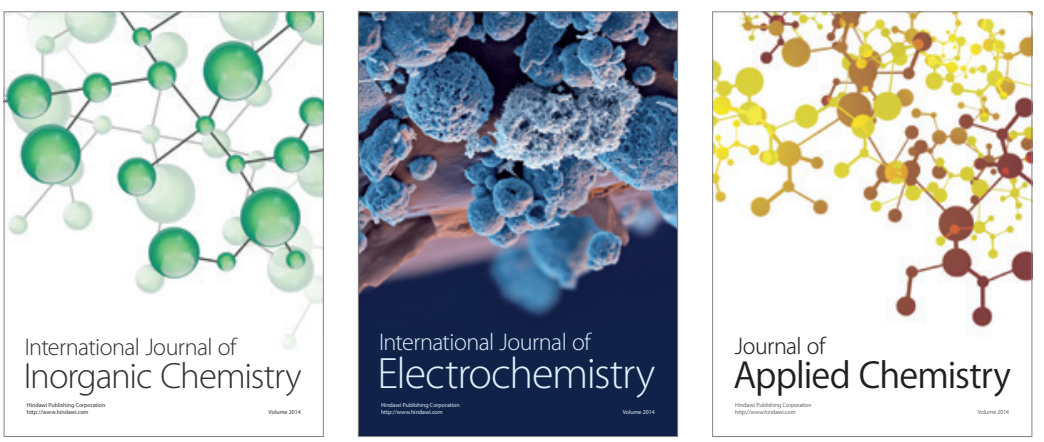

Journal of

Applied Chemistry
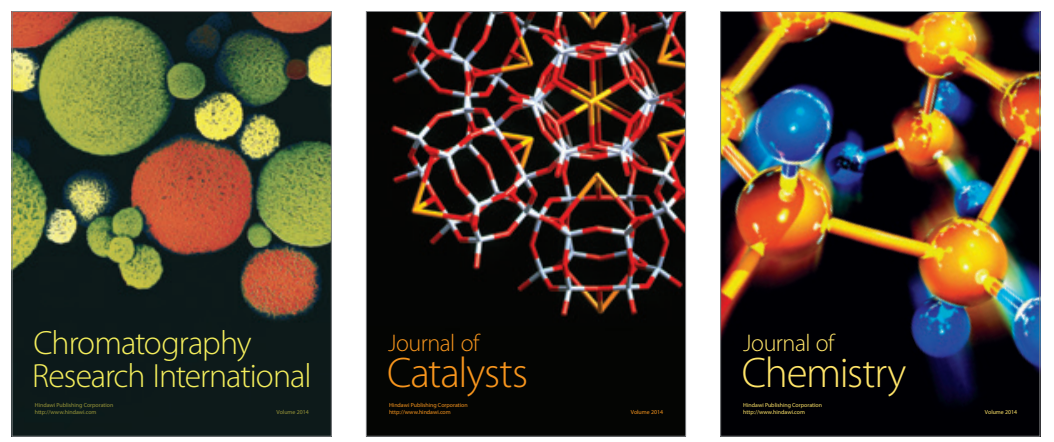
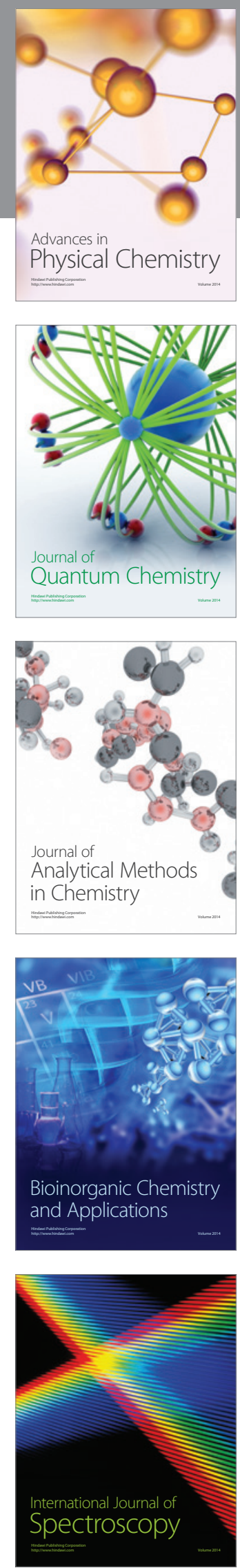